INPLASY

PROTOCOL

To cite: Wang et al. A Bayesian Network Meta-analysis of the Effect of Acute Exercise on Executive Function in Middleaged and Senior People. Inplasy protocol 2021120086. doi:

10.37766/inplasy2021.12.0086

Received: 19 December 2021

Published: 19 December 2021

Corresponding author:

Peng Wang

sus2020rabbit@163.com

Author Affiliation:

Shanghai University of Sport.

Support: 11DZ2261100.

Review Stage at time of this submission: Data analysis.

Conflicts of interest:

None declared.

\section{A Bayesian Network Meta-analysis of the Effect of Acute Exercise on Executive Function in Middle-aged and Senior People}

\author{
Wang, P1; Cai, Z2; Zhao, Q33 Jiang, W4; Liu, C5; Wang, X6.
}

Review question / Objective: Objective: To compare the intervention effect of multiple acute movement formulas on the executive function in middle-aged and senior people and to provide references for the discussion of the plans for precise movements. P: middle-aged and senior people elderly people; I: acute exercise; C: reading or sitting; O: Executive Function; S: RCT/crossover.

Information sources: Randomized searches were carried out in Chinese databases such as CNKI, Wanfang Database, VTTMS, SinoMed and foreign databases such as PubMed, EMBASE, Cochrane Library, Web of Science. The retrieval period is from the beginning of each database to August 2021, supplemented with manual searches for gray literature and references traced back to previous systematic reviews.

INPLASY registration number: This protocol was registered with the International Platform of Registered Systematic Review and Meta-Analysis Protocols (INPLASY) on 19 December 2021 and was last updated on 19 December 2021 (registration number INPLASY2021120086).

\section{INTRODUCTION}

Review question / Objective: Objective: To compare the intervention effect of multiple acute movement formulas on the executive function in middle-aged and senior people and to provide references for the discussion of the plans for precise movements. P: middle-aged and senior people elderly people; I: acute exercise; C: reading or sitting; O: Executive Function; $S$ : $\mathrm{RCT} /$ crossover.

Condition being studied: With the aging problem getting more and more serious, age-associated cognitive decline has already become a global public health issue. The prevalence rates of mild 
cognitive impairment(MCl) are respectively $11.83 \%, 19.23 \%, 24.15 \%, 32.46 \%$ in age groups of 60 69, 70 79, 80 89, 90 years old and above. The prevalence rates of dementia (DE) are 2.89\%, 8.38\%, $14.35 \%$, $31.23 \%$ in these groups. They all increase along with age. $6.7 \%$ of the $\mathrm{MCl}$ patients develop into DE every year. In both diseases, symptoms include varying degrees of lags in response and memory deterioration, impairments in inductive reasoning, spatial orientation and verbal fluency, which seriously influence the quality of life as well as add to the burden of their family and society. Moreover, a slight cognitive decline can lead to an increased risk of death. This explains why the death rate of DE patients is 2.63 times as high as healthy persons'. Irreversible is the cognitive impairment that $\mathrm{MCl}$ and $\mathrm{DE}$ patients already have, so it is in desperate need to find an effective intervention at an early stage in order to prevent or slow the age-associated cognitive decline. One of the main symptoms of age-associated cognitive decline is the decrease in executive function, which is an advanced cognitive process responsible for controlling and regulating other cognitive processes when performing complex cognitive tasks. Many studies have confirmed that exercise can improve cognitive function, especially executive function, in middle-aged and senior people with different cognitive levels, and consequently reduce the risk of developing dementia. Its mechanism may be that exercise increases blood perfusion to the brain and levels of brain-derived neurotrophic factors(BDNF) and insulin-like growth factor-1(IGF-1), stimulates the secretion of catecholamine neurotransmitters (dopamine, norepinephrine, etc.) and promotes the integrity of brain structure, especially the integrity of ectocinerea in the prefrontal and medial temporal lobe regions. It is universally acknowledged that exercise contributes to delaying the cognitive decline in middle-aged and senior people, but influences of the elements (forms, intensity, duration, etc.)in exercise on executive function, which is also called "dosage effect", remain unclear. This hampers the theoretical development and the practical application of the idea that exercise promotes cognitive performance. The research of acute exercise plays a significant part in finding "dosage effect", exploring physiological mechanisms and making long-term and precise exercise formulas, the positive influence of which on executive function has been gradually proved.

\section{METHODS}

Participant or population: middle-aged and senior people elderly people.

Intervention: Acute exercise.

Comparator: Reading or sitting.

Study designs to be included: RCT/ crossover.

Eligibility criteria: Participants are not diagnosed with dementia, neurological disorders or mental health disorder.

Information sources: Randomized searches were carried out in Chinese databases such as CNKI, Wanfang Database, VTTMS, SinoMed and foreign databases such as PubMed, EMBASE, Cochrane Library, Web of Science. The retrieval period is from the beginning of each database to August 2021, supplemented with manual searches for gray literature and references traced back to previous systematic reviews.

Main outcome(s): 30 articles were included (with the sample size of 980 persons). 11 formulas for sports intervention on inhibitory functions are used and 20 25 minutes of aerobic exercise in moderate or high intensity(51\% 70\%HRR), 7 9 practice methods ${ }^{*} 10$ times*2 groups of resistance exercise in moderate intensity(70RM) show remarkable intervention effect. 7 9 practice methods* 10 times ${ }^{\star} 2$ groups of resistance exercise in low intensity(40RM) have inconsistent proofs of intervention effect when direct comparison and indirectly comparison are separately used; 7 formulas for sports intervention on working 
memory are used and resistance exercise in moderate intensity shows remarkable intervention effect; 8 formulas for sports intervention on transfer function are used and 20 25 minutes of aerobic exercise in low and moderate intensity(30\% 60\%HRR) shows remarkable intervention effect, but publication bias may exist. 6 formulas for sports intervention on planning function are used and 20 25 minutes of aerobic exercise in moderate and high intensity (51\% 70\%HRR) shows remarkable intervention effect. Conclusion: Acute exercise has selectively positive impacts on the executive function in middle-aged and senior people. Aerobic exercise in moderate intensity does best in improving the inhibitory, transfer and planning function, while resistance exercise in moderate intensity is most effective in bettering working memory.

Quality assessment / Risk of bias analysis: According to the quality evaluation tool recommended by the Cocharne handbook 5.1 , the methodological quality of the literature included is assessed and the lowquality literature is excluded. The evaluation indexes are the randomized method, allocation concealment, blinded allocation, the integrity of final data, selective reporting, numbers of dislodged cases, follow-up and other biases. There are 3 options for each of them: high risks, low risks and uncertain risks.

Strategy of data synthesis: $R \quad 4.0 .5$ and JAGS 4.3 .0 are used to carry out the model operation and checking. $R \mathbf{4 . 0 . 5}$ is used to draw the ranking chart of cumulative probability (the more raised it is in the upper left part, the better the effect is) and the stacking chart (the higher the rank of effect is, the deeper the color of the bar is). Stata $\mathbf{1 4 . 2}$ is used to draw the network map (sizes of nodes are weighted based on sample capacity, the thickness of links is weighted based on times of comparisons) and the funnel plot. The outcome indicators in this study are all continuous variables, so mean difference(MD) is used as effect size, $95 \%$ confidence interval $(\mathrm{Cl})$ of which not including 0 indicates that the differences are of great significance; based on deviance information criterion (DIC), fixed effect models and random effect models are selected; the Markov Chain Monte Carlo model (MCMC) is used to compare the intervention effects of different acute exercise formulas on the executive function in middle-aged and senior people. Four chains are set up initially for simulation with the iteration step size of 1 and the iteration times of 50000 , the first 20000 of which are annealing to eliminate the impact of initial values; the scale of heterogeneity is quantized by 12 statistics; the nodesplitting method is also used to conduct consistency checks, and if $P>0.05$, it means that there is no obvious difference between direct and indirect comparisons; if the closed-loop structure exists between each intervention measures, global inconsistency tests and local inconsistency tests are required to carry out, using inconsistency factor (IF) and $95 \% \mathrm{Cl}$ to evaluate the consistency of each closed loop. If the value of IF is small and the $95 \% \mathrm{Cl}$ includes 0 , it shows that the loop consistency is relatively excellent; potential scale reduced factor (PSRF) is used to assess the iteration effect of interchain and intrachain variance. If PSRF is close or equal to 1 , it indicates good convergence and stable results; if not, it's necessary to continue using extended arithmetic on this model; surface under the cumulative ranking (SUCRA) is used to reflect the ranking of the effect of each intervention measures, the range of which is $0 \leq$ SUCRA $\leq 100 \%$. The higher the value is, the better the intervention effect is.

Subgroup analysis: According to the partition criterion provided by the guidelines of National Academy of Sports Medicine, the intervention measures mentioned in the documents included are categorized into 13 types; $A=$ control groups (sitting still, reading, chatting, watching videos), $B=10$ minutes of aerobic exercise in low intensity (30\% 50\%HRR), $\mathbf{C}=10$ minutes of aerobic exercise in moderate intensity (51\% 60\%HRR), D= 10 minutes of aerobic exercise in high intensity (61\% 70\%HRR), E= 20 25 minutes of aerobic exercise in low intensity, $F=20 \sim 25$ minutes of aerobic exercise in 
moderate intensity, $G=20 \sim 25$ minutes of aerobic exercise in high intensity, $\mathrm{H}=30 \sim 45$ minutes of aerobic exercise in high intensity, $I=$ 7 9 practice methods*10 times $* 2$ groups of resistance exercise in low intensity(40RM), $J=7 \sim 9$ practice methods*10 times*2 groups of resistance exercise in moderate intensity(70RM), K= 7 9 practice methods ${ }^{*} 10$ times 2 groups of resistance exercise in high intensity (100RM), $L=30$ minutes of aerobic exercise combined with resistance exercise, $M=20$ minutes of intermittent exercise in high intensity.

Sensitivity analysis: None.

Language: No.

Country(ies) involved: American, Japan, China.

Keywords: acute exercise; middle-aged and elderly people; executive function; network meta-analysis.

Contributions of each author:

Author 1 - Peng WANG - Author 1 drafted the manuscript.

Author 2 - Zhidong CAI - Author 2 provides statistical expertise.

Author 3 - Qingying ZHAO.

Author 4 - Wanting JIANG.

Author 5 - Cong LIU.

Author 6 - Xing WANG - Author 6 read, provided feedback, and approved the final manuscript. 